\title{
Adriamycin resistance-associated prohibitin gene inhibits proliferation of human osteosarcoma MG63 cells by interacting with oncogenes and tumor suppressor genes
}

\author{
MIN-DONG DU*, KAI-YI HE* , GANG QIN, JIN CHEN and JIN-YI LI \\ Department of Osteoarthrosis, The First Affiliated Hospital of Guangxi Traditional Chinese Medical University, \\ Nanning, Guangxi 530023, P.R. China
}

Received January 13, 2015; Accepted February 16, 2016

DOI: $10.3892 / \mathrm{ol} .2016 .4862$

\begin{abstract}
The resistance of cancer cells to chemotherapeutic agents is a major obstacle for successful chemotherapy, and the mechanism of chemoresistance remains unclear. The present study developed an adriamycin-resistant human osteosarcoma MG-63 sub-line (MG-63/ADR), and identified differentially expressed proteins that may be associated with adriamycin resistance. Two dimensional gel electrophoresis, matrix-assisted laser desorption ionization time-of-flight mass spectrometry analysis and a protein identification assay were performed. Western blot analysis was used to examine the prohibitin (PHB) levels in the MG-63/ADR cells. Quantitative polymerase chain reaction was utilized to detect adriamycin resistant-associated genes. Laser-scanning confocal microscope was employed to examine the colocalization of PHB with v-myc avian myelocytomatosis viral oncogene homolog (c-myc), FBJ murine osteosarcoma viral oncogene homolog (c-fos), tumor protein p53 and retinoblastoma $1(\mathrm{Rb})$. In addition, the full length of the open reading frame of human PHB was subcloned into a lentiviral vector pLVX-puro. The proliferative rate of MG-63 cells was also investigated. The overall protein expression in MG-63/ADR cells was clearly suppressed. Three notable protein regions, representing high mobility group box 1, Ras homolog gene family, member A, and PHB, were identified to be significantly altered in MG-63/ADR cells when compared with its parental cells. Therefore, PHB modulated the chemoresistance of MG-63/ADR cells by
\end{abstract}

Correspondence to: Professor Gang Qin, Department of Osteoarthrosis, The First Affiliated Hospital of Guangxi Traditional Chinese Medical University, 89-9 Dongge Road, Nanning, Guangxi 530023, P.R. China

E-mail: qingganggx@yeah.net

${ }^{*}$ Contributed equally

Key words: prohibitin, osteosarcoma, MG63 cells, adriamycin resistance-associated genes interacting with multiple oncogenes or tumor suppressor genes (c-myc, c-fos, p53 and Rb). In addition, overexpression of PHB decreases the proliferative rate of MG-63 cells. In conclusion, PHB is an adriamycin resistance-associated gene, which may inhibit the proliferation of human osteosarcoma MG-63 cells by interacting with the oncogenes or tumor suppressor genes, c-myc, c-fos, p53 and $\mathrm{Rb}$.

\section{Introduction}

It has been $\sim 70$ years since chemotherapy was introduced into clinical practice to treat malignant tumors (1). A variety of chemotherapeutic agents have been developed to interfere with the metabolism of cancer cells, including osteosarcoma, colon carcinoma, liver carcinoma and breast carcinoma cells, and a clinician may improve therapeutic outcomes by dose escalation, alterations in the combination of chemotherapy and the addition of irradiation therapy $(1,2)$. However, the overall survival rate of osteosarcoma, colon carcinoma, liver carcinoma and breast carcinoma patients has not improved with the chemotherapeutic agents as much as expected $(1,2)$. Intrinsic and acquired resistance to chemotherapeutic agents is the major obstacle for successful chemotherapy (2). Clinical practice has revealed that the profile of intrinsic gene expression varies greatly in patients that respond poorly to chemotherapy (3). Several genes, including multi-drug resistant protein 1 (4), cluster of differentiation 117 and ATP binding cassette subfamily $\mathrm{G}$ member 2 (5), have been identified as drug resistance genes in human osteosarcoma; however, there is no consensus regarding biomarkers for the detection of cancer resistance to a certain chemotherapy.

Two dimensional gel electrophoresis (2-D PAGE) is a powerful method for analyzing complex protein samples, and previous studies have successfully employed 2-D PAGE for the identification of chemoresistance-associated genes (6). In the present study, an adriamycin-resistant human osteosarcoma MG-63 sub-line was established, and adriamycin resistance-associated proteins were identified by comparing the adriamycin-resistance sub-line with its parental cell line, with the aid of 2-DE and matrix-assisted laser desorption ionization time-of-flight mass spectrometry (MALI-TOF-MS). Out of all the genes that were aberrantly expressed in the resistant 
sub-line, prohibitin (PHB) was demonstrated to be capable of interacting with multiple oncogenes and tumor suppressor genes. This suggests that it has the potential to be a biomarker for chemotherapy resistance.

\section{Materials and methods}

Cell cultures. Human osteosarcoma cell line MG-63 was obtained from the American Type Culture Collection (Manassas, VA, USA). The cells were routinely maintained in complete growth medium [RPMI-1640 (Gibco; Thermo Fisher Scientific, Inc., Waltham, MA, USA) supplemented with $10 \%$ fetal bovine serum (Gibco; Thermo Fisher Scientific, Inc.)] at $37^{\circ} \mathrm{C}$ in a humidified $5 \% \mathrm{CO}_{2}$ incubator. Unless specified, all reagents and materials for cell culture were from Gibco (Thermo Fisher Scientific, Inc.) or Corning Incorporated (Corning, NY, USA).

Establishment of adriamycin resistant MG-63 sub-line. MG-63 cells were routinely maintained in complete growth medium. The method for establishing drug resistant cell lines was performed as previously reported (7). Briefly, the resistant MG-63 cells were established by exposure to increasing concentrations of adriamycin, starting from $4 \mathrm{ng} / \mathrm{ml}$ with a $25 \%$ increase each time. Subsequent to 6 months of continuous cultivation, the subcultures that capable of growing exponentially in the presence of the highest concentration of adriamycin (44 ng/ml) were designated as the adriamycin-resistant sub-line (MG-63/ADR).

2-D-polyacrylamide gel electrophoresis (PAGE), MALDI-TOF-MS analysis and protein identification. 2-D-PAGE was conducted as previously described (8). Briefly, the protein samples from the adriamycin-resistant sub-lines and parental MG-63 cells were diluted in sample buffer with 2\% IPG buffer (pH 3-10; GE Heathcare Life Sciences, Chalfont, UK). The samples were applied to $18-\mathrm{cm}$, immobilized $\mathrm{pH}$ gradient strips (Immobiline Drystrip pH 3-10; GE Healthcare Life Sciences). Upon completion of isoelectric focusing, the strips were equilibrated and the second dimensional electrophoresis was examined overnight at $3 \mathrm{~W} / \mathrm{gel}$ at $20^{\circ} \mathrm{C}$. Triplicate sets of silver-stained gels were scanned using a UMAX POWER LOOK III flat-bed scanner (UMAX Technologies, Dallas, TX, USA) and analyzed with the PDQuest 2-D Analysis software, version 8.0 (Bio-Rad Laboratories, Inc., Hercules, CA, USA). The digitalized 2-DE gel images were compared using an electronic alignment method PDQuest 2-D analysis software version 8.0; Bio-Rad Laboratories, Inc.) (6). Differentially expressed spots were analyzed and annotated.

The spots were cut into pieces and digested with $12.5 \mathrm{ng} / \mu \mathrm{l}$ trypsin (Promega Corporation, Madison, WI, USA) in $50 \mathrm{mM}$ ammonium bicarbonate ( $\mathrm{pH} 8.0$; Sigma-Aldrich, St. Louis, MO, USA). Following elution with $2 \mu \mathrm{l}$ matrix solution consisting of $10 \mathrm{mg} / \mathrm{ml} \alpha$-cyano-3-hydroxy-cinnamic acid (Sigma-Aldrich), the remaining liquid was submitted to a mass spectrometer (MALDI-TOF III; Bruker Corporation, Billerica, MA, USA). The spectra were internally calibrated using the trypsin autolysis products as controls $[842.51 \mathrm{~m} / \mathrm{z}(\mathrm{M}+\mathrm{H})$ and $2,211.11 \mathrm{~m} / \mathrm{z}(\mathrm{M}+\mathrm{H})$ ] by flexImaging software version 2.0 (Bruker Corporation) and blasted against Swiss-Prot (www. uniprot.org/) and National Center for Biotechnology Information BLAST (blast.ncbi.nlm.nih.gov/Blast.cgi) databases using the Proteomics Mascot software version 2.0, which was purchased from Matrix Science, Inc. (Boston, MA, USA). All searches were analyzed with a 50 ppm mass tolerance.

Western blot analysis. For immunoblot analysis, the MG-63/ADR and parental MG-63 cells were washed with phosphate-buffered saline, and subsequently lysed with radioimmunoprecipitation buffer (Tiangen Biotech Co., Ltd., Beijing, China). Following centrifugation at 12,000 x g for $15 \mathrm{~min}$ at $4^{\circ} \mathrm{C}$, the supernatant was separated on a $10 \%$ sodium dodecyl sulfate-PAGE gel (Tiangen Biotech Co., Ltd.) and transblotted onto a polyvinylidene difluoride membrane (Sigma-Aldrich). Following blocking with $5 \%$ bovine serum albumin (BSA; Sigma-Aldrich) in Tris-buffered saline and Tween 20, the membrane was incubated with mouse antihuman PHB monoclonal antibody (catalogue no. sc-377037; 1:3,000; Santa Cruz Biotechnology, Inc., Dallas, TX, USA) at $4^{\circ} \mathrm{C}$ overnight, and then with goat anti-mouse immunoglobulin (Ig) G polyclonal antibody (catalogue no. sc-395763; 1:2,000; Santa Cruz Biotechnology, Inc.) at $37^{\circ} \mathrm{C}$ for $2 \mathrm{~h}$. Specific bands were visualized using Odyssey ${ }^{\circledR}$ CLx Imaging System (LI-COR Biotechnology, Lincoln, NE, USA). $\beta$-actin was used as an indicator for quality of protein loading.

Reverse transcription- quantitative polymerase chain reaction (RT-qPCR) analysis of adriamycin resistance-associated genes. The RNA from MG-63/ADR and parental MG-63 cells was isolated using TRIzol ${ }^{\circledR}$ Reagent (catalog no., D9108A; Takara Bio, Inc., Otsu, Japan), according to the manufacturer's protocol. cDNA samples were prepared using a 1st Strand cDNA Synthesis kit (catalog no., D6110S; Takara Bio, Inc.), according to the manufacturer's protocol. The primer pairs for qPCR were as follows: $\beta$-actin, forward 5'-ACATCT GCTGGAAGGTGGAC-3' and reverse 5'-CTGTGGCAT CCACGAAACTA-3'; and PHB, forward 5'-CGGAGAGGA CTATGATGAG-3' and reverse 5'-GGTCAGATGTGTCAA GGA-3' (Sangon Biotech Co., Ltd., Shanghai, China). qPCR was performed using SYBR ${ }^{\circledR}$ Premix Ex Taq ${ }^{\mathrm{TM}}$ II kit (catalog no., RR820A; Takara, Bio, Inc.) in ABI PRISM ${ }^{\circledR} 7900 H T$ Sequence Detection System (Applied Biosystems ${ }^{\circledR}$; Thermo Fisher Scientific, Inc.). Hot-start PCR was performed as follows: $30 \mathrm{sec}$ at $95^{\circ} \mathrm{C}$; 45 cycles, with 1 cycle of $5 \mathrm{sec}$ at $95^{\circ} \mathrm{C}$ and 44 cycles of $30 \mathrm{sec}$ at $60^{\circ} \mathrm{C}$. All samples were read in triplicate, and values were normalized to $\beta$-actin expression. The relative expression data were calculated according to the $2^{-\Delta \mathrm{Cq}}$ method and presented as fold-change (7).

Laser-scanning confocal microscope. The cells were seeded on cover slips overnight prior to the following experiment. Subsequent to fixation with $3 \%$ paraformaldehyde (Sigma-Aldrich) in PBS ( $\mathrm{pH} \mathrm{7.4)} \mathrm{for} 10 \mathrm{~min}$ at room temperature, the cover slips were washed in ice-cold PBS three times. Subsequently, the cover slips were immersed in PBS containing $0.25 \%$ Triton X-100 for 10 min to permeabilize cell membranes. Following washing in PBS three times for $5 \mathrm{~min}$ for each wash, the cells were incubated with $1 \%$ BSA in PBS and $0.05 \%$ Tween 20 (PBST) for $30 \mathrm{~min}$ at room temperature to block the unspecific binding of the antibodies. Subsequently, the 
Table I. Differentially expressed proteins in adriamycin-resistant human osteosarcoma MG-63 and parental MG-63 cells.

\begin{tabular}{lccccc}
\hline Spot no. & Protein & NCBI ID & Theoretical isoelectric point, pI & Theoretical Mw, kDa & Expression intensity \\
\hline S1 & HMGB1 & GI:48145843 & 8.20 & 25.4 & High \\
S2 & RhoA & GI:6706217 & 5.73 & 24.9 & High \\
S3 & Prohibitin & GI:246483 & 5.76 & 29.8 & Low
\end{tabular}

Genes were identified using matrix-assisted laser desorption ionization time-of-flight mass spectrometry. HMGB1, high mobility group box 1 protein; RhoA, Ras homolog gene family, member A; NCBI, National Center for Biotechnology Information; Mw, molecular weight.

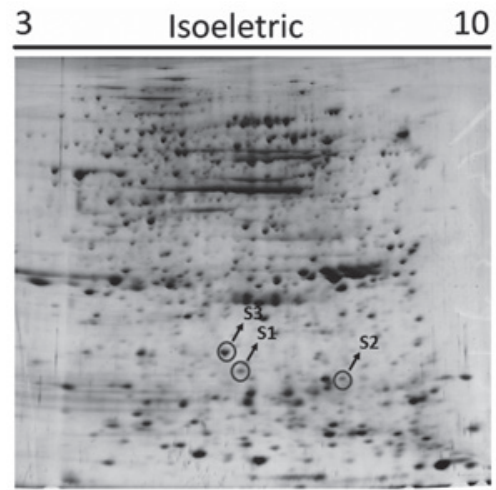

Parental MG-63 cells

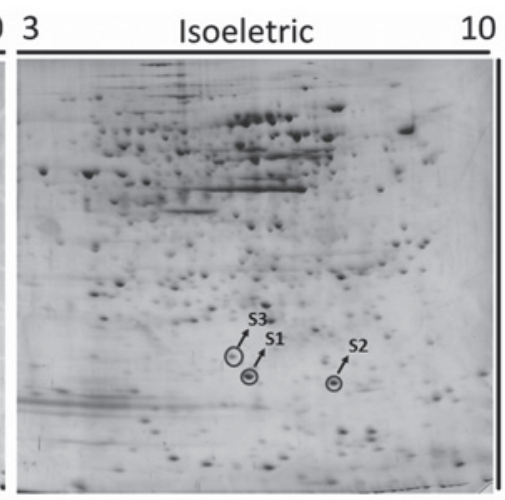

Resistant MG-63 cells

Figure 1. Representative image of silver-stained two dimensional gel electrophoresis map of human osteosarcoma parental MG-63 and adriamycin-resistant MG-63 cells. S1, high mobility group box 1 protein; S2, Ras homolog gene family, member A; S3, prohibitin.

cells were incubated in the mixture of two primary antibodies [mouse anti-human PHB (monoclonal antibody; catalogue no. sc-377037; 1:2,000; Santa Cruz Biotechnology, Inc.)/rabbit anti-human FBJ murine osteosarcoma viral oncogene homolog (c-fos; polyclonal antibody; catalogue no. sc-52; 1:2,000; Santa Cruz Biotechnology, Inc.); mouse anti-human PHB (monoclonal antibody; catalogue no. sc-377037; 1:2,000; Santa Cruz Biotechnology, Inc.)/rabbit anti-human v-myc avian myelocytomatosis viral oncogene homolog (c-myc; polyclonal antibody; catalogue no. sc-788; 1:2,000; Santa Cruz Biotechnology, Inc.); mouse anti-human PHB (monoclonal antibody; catalogue no. sc-377037; 1:2,000; Santa Cruz Biotechnology, Inc.)/rabbit anti-human tumor protein p53 (polyclonal antibody; catalogue no. sc-6243; 1:2,000; Santa Cruz Biotechnology, Inc.); and mouse anti-human PHB (monoclonal antibody; catalogue no. sc-377037; 1:2,000; Santa Cruz Biotechnology, Inc.)/rabbit anti-human retinoblastoma $1(\mathrm{Rb}$; monoclonal antibody; catalogue no. sc-1538; 1:2,000; Santa Cruz Biotechnology, Inc.)] in $1 \% \mathrm{BSA}$ in PBST in a humidified chamber for $1 \mathrm{~h}$ at room temperature. Following washing 3 times in PBS for $5 \mathrm{~min}$, the cells were incubated with the mixture of two secondary antibodies [cyanine dye $\left(\mathrm{Cy}^{\circledR} 3\right)$-conjugated goat anti-mouse IgG (polyclonal antibody; catalogue no. 115-165-164; 1:2,000; Jackson ImmunoResearch Laboratories, Inc., West Grove, PA, USA)/Cy3-conjugated goat anti-rabbit IgG (polyclonal antibody; catalogue no. 111-165-003; 1:2,000; Jackson ImmunoResearch Laboratories, Inc.)] in $1 \%$ BSA for $1 \mathrm{~h}$ at room temperature in dark. The cells were washed in PBS for $5 \mathrm{~min}$ in the dark. For counter staining, the cells were incubated in DAPI (Sigma-Aldrich) for $5 \mathrm{~min}$ at $37^{\circ} \mathrm{C}$. Following rinsing in
PBS, the cells were mounted with a drop of mounting medium (Gibco; Thermo Fisher Scientific, Inc.) and sealed with nail polish to prevent movement under the microscope. Image acquisition was performed by laser confocal scanning microscopy (TCS-SP2 MP; Leica Microsystems, Inc., Buffalo Grove, IL, USA).

Overexpression of $P H B$ in $M G-63 / A D R$ sub-line. The full length of open reading frame of human PHB was subcloned into lentiviral vector pLVX-puro (Invitrogen; Thermo Fisher Scientific, Inc.). The lentivirus was subsequently generated by cotransfection of human embryonic kidney 293T cells with the recombinant lentiviral expression vector and Lenti- $\mathrm{X}^{\mathrm{TM}}$ Packaging System from Clontech Laboratories, Inc. (Mountainview, CA, USA). For probing the effects of PHB on cell growth, the resistant MG-63/ADR sub-line was transduced with lentivirus bearing PHB (Invitrogen; Thermo Fisher Scientific, Inc.), and a stable clone were screened by limiting dilution under the selective pressure of puromycin $(2.5 \mu \mathrm{g} / \mathrm{ml}$; Sigma-Aldrich). Cell growth curve analysis was performed as described previously (9).

3-(4,5-dimethylthiazol-2-yl)-2,5-diphenyltetrazolium bromide (MTT) assay. Cells were cultured in 96-well tissue culture plates. At 24 and $48 \mathrm{~h}$ after transfection, MTT (Sigma-Aldrich) was added to each well to a final concentration of $5 \mathrm{mg} / \mathrm{ml}$ in culture medium, and incubated at $37^{\circ} \mathrm{C}$ for $4 \mathrm{~h}$. The reaction was terminated by removal of the supernatant and addition of $150 \mu \mathrm{l}$ dimethyl sulfoxide (Sigma-Aldrich) to dissolve the formazan product. The plates were read at $405 \mathrm{~nm}$ on an MK3 


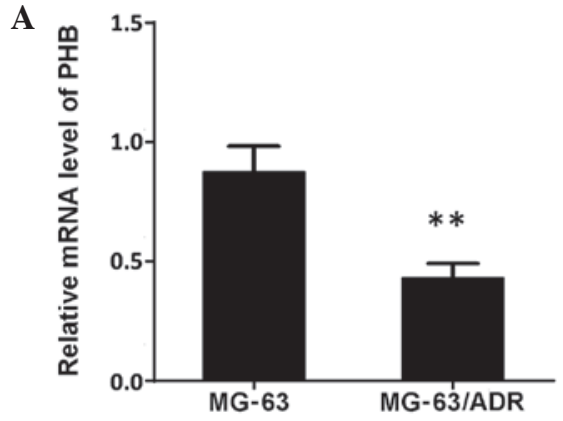

B

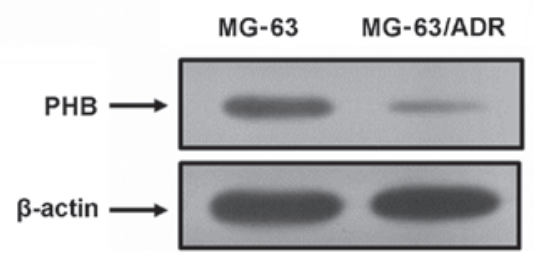

Figure 2. Aberrant expression of PHB in MG-63/ADR and human osteosarcoma MG-63 cells. (A) Quantitative polymerase chain analysis for mRNA expression of PHB. Results are representative of 3 experiments. ${ }^{* *} \mathrm{P}<0.01$, PHB mRNA expression in MG-63/ADR cells vs. MG-63 cells. (B) Western blot analysis for PHB expression. PHB, prohibitin; MG-63/ADR, human osteosarcoma adriamycin-resistant cells; mRNA, messenger RNA.

$\mathbf{A}$
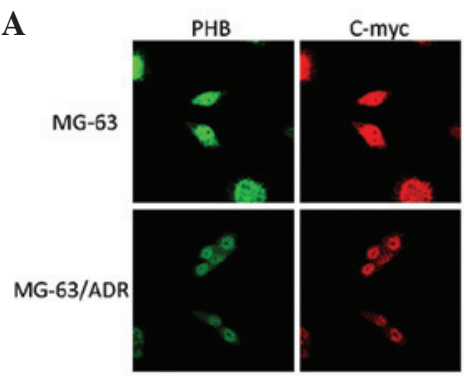

${ }_{\text {MG-63 }}$

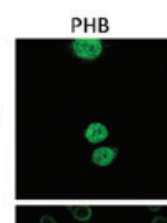

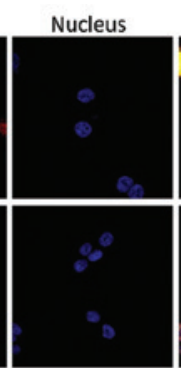
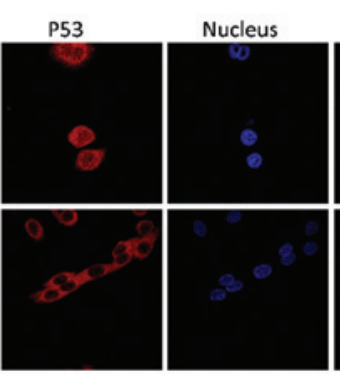

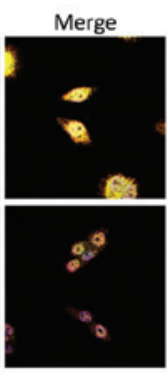

B
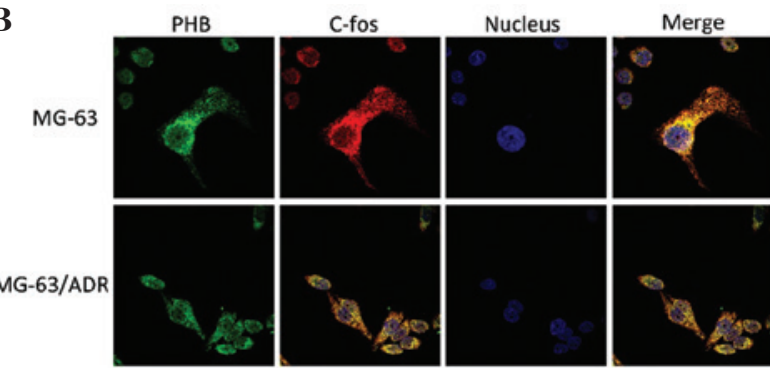

MG-63/ADR
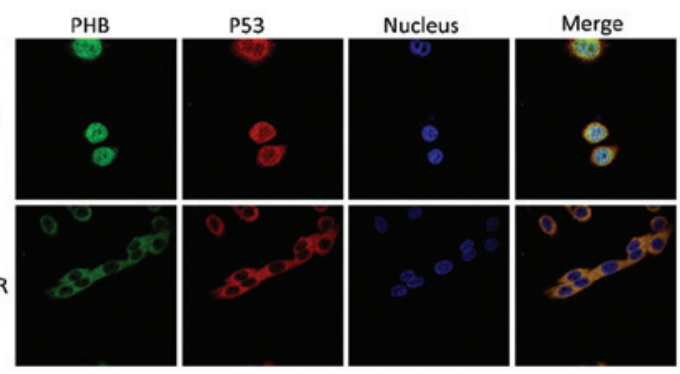

D
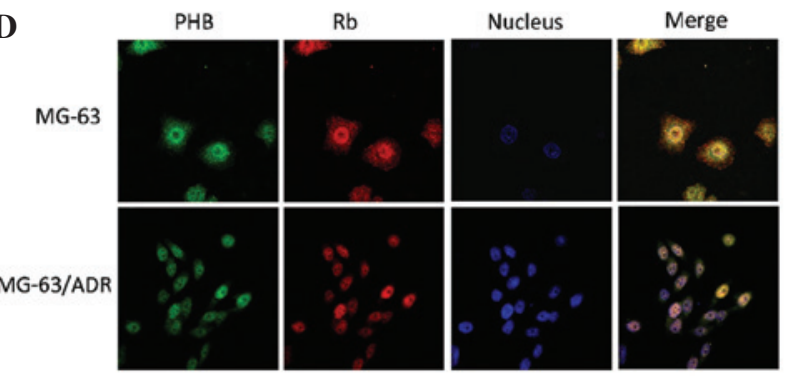

Figure 3. Colocalization of PHB with c-myc, c-fos, p53 and Rb in human osteosarcoma MG-63 and MG-63/ADR cells. Colocalization of PHB with (A) c-myc, (B) c-fos, (C) p53 and (D) Rb. MG-63/ADR, human osteosarcoma adriamycin-resistant cells; PHB, prohibitin; c-myc, v-myc avian myelocytomatosis viral oncogene homolog; c-fos, FBJ murine osteosarcoma viral oncogene homolog; p53, tumor protein p53; Rb, retinoblastoma 1.

micro-ELISA plate reader (Thermo Fisher Scientific, Inc.). Each assay was performed in duplicates of 10 wells.

Statistical analysis. SPSS 19.0 software (IBM SPSS, Armonk, NY, USA) was used to perform statistical analysis. Data are represented as the mean, median, minimum and maximum values. Student's t test was used to analyze the data. $\mathrm{P}<0.05$ was considered to indicate a statistically significant difference.

\section{Results}

Proteomic analysis of MG-63 cells pre-and post-chemoresistance. The whole cell lysates from MG-63 and MG-63/ADR cells were separated using 2-DE, and the gel was visualized by silver-staining. The procedure was independently repeated 3 times and a representative gel image is shown in Fig. 1. The number of protein spots in the MG-63/ADR sub-line markedly decreased when compared with the parental MG-63 cells. The spots differentiated by intensity were excised and digested with trypsin, and were subsequently identified by mass spectrometry. The identified proteins are listed in Table I.

Analysis of aberrant expression of $P H B$ by $q P C R$ and western blot analysis. To additionally verify the aberrant alterations identified by 2D-PAGE, western immunoblotting and RT-qPCR were employed to confirm expression levels. The results showed that PHB in MG-63/ADR cells was much lower compared with parental MG-63 cells, suggesting the expression of PHB in the MG-63/ADR cells was significantly inhibited (Fig. 2; $\mathrm{P}<0.01$ ). These results were consistent with the 2-DE analysis.

Colocalization of PHB with $c-m y c, c-f o s, p 53$ and Rb staining. The MG-63 and MG-63/ADR cells were immunostained with primary antibodies for PHB, c-myc, c-fos, p53 and Rb. The anti-PHB antibody and other antibodies were labeled with red fluorescence $\mathrm{Cy} 3$. Laser confocal scanning microscopy was used to observe the alteration of colocalization of PHB with other proteins. The colocalized region was yellow or orange. 
A

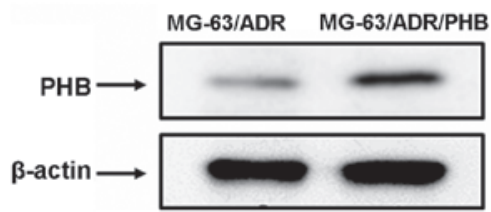

B

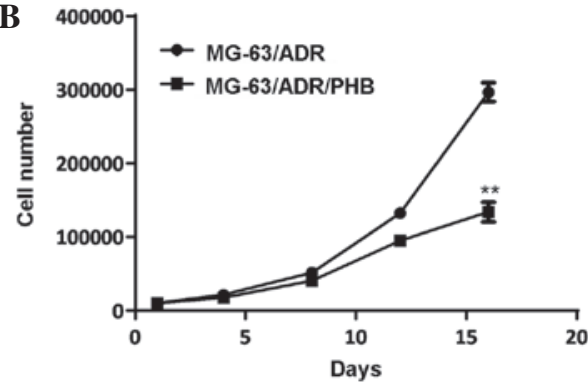

Figure 4. Overexpression of PHB in MG-63/ADR cells decreases the proliferative rate of the cells. (A) Western blot analysis for PHB expression. (B) Proliferative rate of MG-63/ADR and MG-63/ADR/PHB cells. The proliferative rate was calculated according to the cell count method. ${ }^{* * *} \mathrm{P}<0.01$, cell number in MG-63/ADR/PHB cells vs. MG-63/ADR group. PHB, prohibitin; MG-63/ADR, human osteosarcoma adriamycin-resistant cells; MG-63/ADR/PHB, human osteosarcoma adriamycin-resistant cells overexpressing PHB.

Colocalization of PHB with c-myc. The green fluorescence representing PHB was distributed throughout the MG-63 cells. The fluorescent density in the nucleus was clearer compared with the cytoplasm. The red fluorescence representing c-myc was markedly distributed in the nucleus and the density was not uniform. In images where the red and green fluorescence has been merged together, PHB was observed to be colocalized with c-myc in the nucleoplasm region, particularly in the nucleolus region (Fig. 3A). However, in the MG-63/ADR cells, the PHB expression was primarily distributed in nucleus, and the red fluorescence of c-myc was decreased and uniformly distributed in the nucleus. The merged images demonstrated that the colocalization of PHB with c-myc in the cytoplasm was not clear, suggesting the colocalized region of PHB with c-myc was not in the cytoplasm (Fig. 3A).

Colocalization of PHB with c-fos. In MG-63 parental cells, PHB and c-fos were primarily expressed in the cytoplasm and nucleoplasm expression was extremely weak. PHB and c-fos colocalized together in the cytoplasm; however, the overall fluorescent intensity in MG-63/ADR cells was much lower compared with parental MG-63 cells, indicating that PHB and c-fos were downregulated when the MG-63 cells were conferred with adriamycin resistance. Nevertheless, the colocalization region was not altered pre- and post-chemoresistance (Fig. 3B).

Colocalization of PHB with p53. Cytoplasmic p53 was dominant in parental MG-63 cells. The fluorescence intensity of PHB and p53 was relatively weak in the nucleus. The expression of PHB and p53 was entirely attenuated in MG-63/ADR cells, and the fluorescence in the nucleus was almost completely depleted. However, the colocalization of PHB with p53 was present in cells pre- and post-chemoresistance (Fig. 3C).

Colocalization of PHB with Rb. The green fluorescence representing PHB was distributed in the nucleus region of parental MG-63 cells. The fluorescent density in the nucleolus was relatively clear, while the fluorescence in the cytoplasm was much weaker. The red fluorescence representing $\mathrm{Rb}$ was distributed throughout the whole cell. The merged image indicated that PHB colocalized with $\mathrm{Rb}$ in the nucleoplasm region, particularly in the nucleolus region (Fig. 3D). Adriamycin treatment did not affect the colocalization of PHB with $\mathrm{Rb}$, but did attenuated their expression levels.

Overexpression of $P H B$ in $M G-63 / A D R$ cells. To additionally elucidate the function of PHB in modulating the sensitivity of MG-63 cells to chemotherapeutic drugs, the expression of PHB was artificially increased in the MG-63/ADR cells using a lentiviral expression vector (Fig. 4A). The cell proliferation assay revealed that overexpression of PHB decreased the proliferative rate of MG-63/ADR cells (Fig. 4B; $\mathrm{P}<0.01$ ), indicating its pivotal role in mediating chemoresistance in human osteosarcoma cells.

\section{Discussion}

Chemotherapy has prolonged the life span of patients with localized osteosarcoma $(10,11)$. However, almost one third of patients with localized osteosarcoma suffer from recurrence or progressive disease due to the development of drug resistance $(10,11)$. The present study developed an adriamycin-resistant MG-63 sub-line, and employed the 2-DE method to identify differentially expressed genes in the resistant sub-line compared with the parental MG-63 cells. All these differentially expressed genes are directly associated with chemoresistance, and one of these, PHB, is involved in the evolution of osteosarcoma resistance to adriamycin.

RhoA is a member of the Ras superfamily, which regulates cytoskeletal dynamics; therefore participating in multiple cellular activities, including cell motility and polarity (12). The Rho subfamily includes three isoforms RhoA, RhoB and RhoC, which share $84 \%$ homology in sequence differing near the $\mathrm{C}$ terminus (13). When overexpressed, RhoA, RhoB and RhoC induce stress fibers and induce terminal morphological alterations during apoptosis (14). However, several studies have indicated that the three isoforms have distinct functions. RhoA is localized to the plasma membrane, while RhoB is directed to endosomal membranes, due to its unique $\mathrm{C}$-terminal lipid modifications, and manipulates the endosomal trafficking of membrane receptors (15). Furthermore, RhoA inhibits cancer cell invasion in vitro, whereas RhoC often enhances cancer metastasis (16). Depletion of RhoA promotes cell invasion (17) and constitutive overexpression in $\mathrm{T}$ cells induces the expression of anti-apoptotic protein B-cell lymphoma-2, which protects cells from apoptosis (18). By contrast, knockdown of RhoA results in the apoptosis of T cells (19). The present data demonstrates that the expression levels of RhoA were markedly increased in the MG-63/ADR sub-line, which may have a role in overcoming cytotoxic drug-induced apoptotic cell death. 
High-mobility group box 1 (HMGB1) is a highly conserved nuclear protein, which is a damage-associated molecule that interacts with receptors for advanced glycation end products and toll-like receptors (20-22). A number of studies have revealed its pivotal role in mediating autophagy in cancer development and therapy $(23,24)$. Endogenous HMGB1 may negatively regulate apoptosis of tumor cells, and manipulating HMGB1 expression may alter the sensitivity of cancer cells to chemotherapeutic drugs $(25,26)$. Various anticancer agents, including doxorubicin, cisplatin and methotrexate, upregulate HMGB1 expression in human osteosarcoma cells, while suppression of its expression using RNA interference-mediated knockdown restores the chemosensitivity of osteosarcoma cells in vivo and in vitro $(27,28)$. The present data demonstrated that HMGB1 levels were increased in the MG-63/ADR sub-line, which was consistent with the results from another study (28), indicating it may serve as a candidate gene for monitoring osteosarcoma chemoresistance.

PHB is known as a tumor suppressor and is ubiquitously expressed in multiple tissues with antiproliferative properties (29). It controls the transition from G1 to $S$ phase in cycling cells (29). High levels of PHB are commonly observed in various human cancer solid tumor cell lines $(30,31)$. In the nucleus, PHB interacts with E2F transcription factor 1 (32), p53 and phosphorylated $\mathrm{Rb}$ (33) to regulate the expression of genes that are associated with cell proliferation and differentiation. The present data demonstrated that the level of PHB in the MG-63/ADR sub-line was decreased compared to parental cells. In addition, the present data from laser confocal microscopy revealed that PHB colocalized with c-myc, c-fos, p53 and $\mathrm{Rb}$ in the parental MG-63 cells; however, the regions where colocalization was observed was distinct from colocalization regions observed in the MG-63/ADR sub-line. Furthermore, the fluorescence intensity representing PHB staining was attenuated in the resistant sub-line compared with the parental MG-63 cells. Overexpression of PHB in the MG-63/ADR sub-line decreased the proliferative rate of cells in the present study. Previously, it was observed that a deletion of the PHB gene led to an $80 \%$ reduction of mitochondrial potential (34), and subsequently triggered the release of apoptogenic factors, indicating that PHB-regulated mitochondria potential may also affect chemotherapeutic effects.

Overall, the present study employed 2-DE and MALDI-TOF-MS methods and identified notable genes that respond to adriamycin resistance in human osteosarcoma cells. The functions of these genes were associated with apoptotic signaling pathways. Of all the identified genes, PHB was demonstrated to be a promising target for novel therapeutic strategies, as it interacted with c-myc, c-fos, p53 and Rb, and an overexpression of PHB modulated the proliferative rate of adriamycin-resistant MG-63 cells. However, additional study is required to elucidate how these chemoresistance-associated genes interfere with the adriamycin-activated pathway leading to adriamycin resistance in human osteosarcoma.

\section{Acknowledgements}

The present study was supported by the National Natural Science Foundation of China (Beijing, China; grant no., 81360550).

\section{References}

1. Saleh EM, El-Awady RA and Anis N: Predictive markers for the response to 5-fluorouracil therapy in cancer cells: Constant-field gel electrophoresis as a tool for prediction of response to 5-fluorouracil-based chemotherapy. Oncol Lett 5: 321-327, 2013.

2. Lewis IJ, Nooij MA, Whelan J, Sydes MR, Grimer R, Hogendoorn PC, Memon MA, Weeden S, Uscinska BM, van Glabbeke M, et al: Improvement in histologic response but not survival in osteosarcoma patients treated with intensified chemotherapy: A randomized phase III trial of the European osteosarcoma intergroup. J Natl Cancer Inst 99: 112-128, 2007.

3. MintzMB,Sowers R, Brown KM,Hilmer SC,Mazza B,Huvos AG, Meyers PA, Lafleur B, McDonough WS, Henry MM, et al: An expression signature classifies chemotherapy-resistant pediatric osteosarcoma. Cancer Res 65: 1748-1754, 2005.

4. Scotlandi K, Serra M, Nicoletti G, Vaccari M, Manara MC, Nini G, Landuzzi L, Colacci A, Bacci G, Bertoni F, et al: Multidrug resistance and malignancy in human osteosarcoma. Cancer Res 56: 2434-2439, 1996.

5. Adhikari AS, Agarwal N, Wood BM, Porretta C, Ruiz B, Pochampally RR and Iwakuma T: CD117 and Stro-1 identify osteosarcoma tumor-initiating cells associated with metastasis and drug resistance. Cancer Res 70: 4602-4612, 2010.

6. Zhou J, Wei YH, Liao MY, Xiong Y, Li JL and Cai HB: Identification of cisplatin-resistance associated genes through proteomic analysis of human ovarian cancer cells and a cisplatin-resistant subline. Asian Pac J Cancer Prev 13: 6435-6439, 2012.

7. Zhang X, Yashiro M, Qiu H, Nishii T, Matsuzaki T and Hirakawa K: Establishment and characterization of multidrug-resistant gastric cancer cell lines. Anticancer Res 30: 915-921, 2010.

8. Görg A, Drews O, Lück C, Weikand F and Weiss W: 2-DE with IPGs. Electrophoresis 30 (Suppl 1): S122-S132, 2009.

9. Dannenberg JH, David G, Zhong S, van der Torre J, Wong WH and Depinho RA: mSin3A corepressor regulates diverse transcriptional networks governing normal and neoplastic growth and survival. Genes Dev 19: 1581-1595, 2005.

10. Chen Y, Yang Y, Yuan Z, Wang C and Shi Y: Predicting chemosensitivity in osteosarcoma prior to chemotherapy: An investigational study of biomarkers with immunohistochemistry. Oncol Lett 3: 1011-1016, 2012.

11. Goorin AM, Schwartzentruber DJ, Devidas M, Gebhardt MC, Ayala AG, Harris MB, Helman LJ, Grier HE and Link MP; Pediatric Oncology Group: Presurgical chemotherapy compared with immediate surgery and adjuvant chemotherapy for nonmetastatic osteosarcoma: Pediatric oncology group study POG-8651. J Clin Oncol 21: 1574-1580, 2003.

12. Jaffe AB and Hall A: Rho GTPases: Biochemistry and biology. Annu Rev Cell Dev Biol 21: 247-269, 2005.

13. Wheeler AP and Ridley AJ: Why three Rho proteins? RhoA, RhoB, RhoC and cell motility. Exp Cell Res 301: 43-49, 2004.

14. Aznar S and Lacal JC: Rho signals to cell growth and apoptosis. Cancer Lett 165: 1-10, 2001.

15. Adamson P, Paterson HF and Hall A: Intracellular localization of the P21rho proteins. J Cell Biol 119: 617-627, 1992.

16. Simpson KJ, Dugan AS and Mercurio AM: Functional analysis of the contribution of RhoA and RhoC GTPases to invasive breast carcinoma. Cancer Res 64: 8694-8701, 2004.

17. Vega FM, Fruhwirth G, Ng T and Ridley AJ: RhoA and RhoC have distinct roles in migration and invasion by acting through different targets. J Cell Biol 193: 655-665, 2011.

18. Cho HJ, Baek KE, Park SM, Kim IK, Nam IK, Choi YL, Park SH, Im MJ, Choi J, Ryu J, et al: RhoGDI2 confers gastric cancer cells resistance against cisplatin-induced apoptosis by upregulation of Bcl-2 expression. Cancer Lett 311: 48-56, 2011.

19. Costello PS, Cleverley SC, Galandrini R, Henning SW and Cantrell DA: The GTPase rho controls a p53-dependent survival checkpoint during thymopoiesis. J Exp Med 192: 77-85, 2000.

20. Lotze MT and Tracey KJ: High-mobility group box 1 protein (HMGB1): Nuclear weapon in the immune arsenal. Nat Rev Immunol 5: 331-342, 2005.

21. Wang W, Jiang H, Zhu H, Zhang H, Gong J, Zhang L and Ding Q: Overexpression of high mobility group box 1 and 2 is associated with the progression and angiogenesis of human bladder carcinoma. Oncol Lett 5: 884-888, 2013.

22. Cunha C, Carvalho A, Esposito A, Bistoni F and Romani L: DAMP signaling in fungal infections and diseases. Front Immunol 3: 286, 2012. 
23. White E and DiPaola RS: The double-edged sword of autophagy modulation in cancer. Clin Cancer Res 15: 5308-5316, 2009.

24. Livesey KM, Tang D, Zeh HJ and Lotze MT: Autophagy inhibition in combination cancer treatment. Curr Opin Investig Drugs 10: 1269-1279, 2009

25. Xie M, Kang R, Yu Y,Zhu S, He YL, Xu WQ, Tang DL and Cao LZ Enhancive effect of HMGB1 gene silence on adriamycin-induced apoptosis in K562/A02 drug resistance leukemia cells. Zhonghua Xue Ye Xue Za Zhi 29: 549-552, 2008 (In Chinese).

26. Yu Y, Xie M, He YL, Xu WQ, Zhu S and Cao LZ: Role of high mobility group box 1 in adriamycin-induced apoptosis in leukemia K562 cells. Ai Zheng 27: 929-933, 2008 (In Chinese).

27. Huang J, Ni J, Liu K, Yu Y, Xie M, Kang R, Vernon P, Cao L and Tang D: HMGB1 promotes drug resistance in osteosarcoma. Cancer Res 72: 230-238, 2012.

28. Huang J, Liu K, Yu Y, Xie M, Kang R, Vernon P, Cao L, Tang D and Ni J: Targeting HMGB1-mediated autophagy as a novel therapeutic strategy for osteosarcoma. Autophagy 8: 275-277, 2012.
29. Dell'Orco RT, McClung JK, Jupe ER and Liu XT: Prohibitin and the senescent phenotype. Exp Gerontol 31: 245-252, 1996.

30. Coates PJ, Nenutil R, McGregor A, Picksley SM, Crouch DH Hall PA and Wright EG: Mammalian prohibitin proteins respond to mitochondrial stress and decrease during cellular senescence. Exp Cell Res 265: 262-273, 2001.

31. Czarnecka AM, Campanella C, Zummo G and Cappello F: Mitochondrial chaperones in cancer: From molecular biology to clinical diagnostics. Cancer Biol Ther 5: 714-720, 2006.

32. Rizwani W, Alexandrow $M$ and Chellappan S: Prohibitin physically interacts with MCM proteins and inhibits mammalian DNA replication. Cell Cycle 8: 1621-1629, 2009.

33. Zhou TB and Qin YH: Signaling pathways of prohibitin and its role in diseases. J Recept Signal Transduct Res 33: 28-36, 2013.

34. Gogvadze V, Orrenius S and Zhivotovsky B: Mitochondria as targets for cancer chemotherapy. Semin Cancer Biol 19: 57-66, 2009. 